\title{
Development of Hybrid-Anvil-Cell for Polarized and Unpolarized Neutron Diffraction Study
}

\author{
Noriki Terada $^{1 *}$ \\ 1 National Institute for Materials Science, 1-2-1, Sengen, Tsukuba, Ibaraki, 305-0047, Japan \\ * Corresponding author: Fax: 81-29-859-2801, e-mail: terada.noriki@nims.go.jp
}

\begin{abstract}
We developed a hybrid anvil high-pressure cell (HAC) for neutron diffraction experiments with multi-extreme conditions, including high pressure, a high magnetic field, and low temperature, and for spherical neutron polarimetry analysis under pressure. By using the HAC, we successfully conducted neutron diffraction experiments under high-pressure $(5.0 \mathrm{GPa})$ and high-magnetic-field $(8 \mathrm{~T})$ conditions, and conducted spherical neutron polarimetry analysis at up to $4.0 \mathrm{GPa}$. This review introduces the technical development of the HAC and recent experimental results for multiferroic materials.

Key words: high-pressure, neutron diffraction, multiferroics, spherical neutron polarimetry
\end{abstract}

\section{Introduction}

Neutron diffraction is one of the most powerful techniques for investigating magnetic ordering. However, neutron experiments require a relatively large sample volume compared with that for other techniques, such as $\mathrm{X}$-ray diffraction. This is a serious drawback for high-pressure experiments, for which sample size is limited to a small volume.[1,2] Experiments with a combination of high pressure, low temperature, and high magnetic field are required for understanding physical properties in such extreme conditions in condensed matter physics. Although high-pressure neutron diffraction experiments have many challenges, some high-pressure devices have been developed for experiments beyond $10 \mathrm{GPa}$ in some neutron facilities.

For example, the Paris-Edinburgh (PE) press has been widely used in some European neutron facilities for a long time.[2] The PE press has a relatively large sample volume, on the order of several cubic millimeters, making it possible to measure powder samples as well as single-crystal samples. Sapphire anvil cells have been developed mainly in Laboratory Leon Brillouin, France. The cell can be used for low-temperature and applied magnetic field conditions because of its compact size.[1] Osakabe developed the hybrid anvil cell (HAC), which utilizes an anvil made of two materials, namely WC and sapphire (or $\mathrm{SiC}$ ) crystals.[3,4] The cell has been used for unpolarized neutron diffraction experiments at pressures of up to $10 \mathrm{GPa}$.

Several studies have recently been performed using the HAC. (i) Combination of HAC and the cold neutron time of flight neutron instrument WISH at ISIS neutron facility. Using this combination, one can detect a wide reciprocal lattice space at the same time because of the large opening windows of the HAC, $[3,4]$ the wide coverage of the neutron detectors, and the wide energy distribution of incident neutrons on WISH time-of-flight (TOF) diffractometry.[5] (ii) Non-magnetic HAC for spherical neutron polarimetry (SNP) analysis experiment with CRYOgenic Polarization Analysis Device (CRYOPAD) on D3 beamline at Institut Laue Langevin (ILL). By using SNP analysis, [6] one can determine the magnetic structure with precise spin orientation even for a small number of observable magnetic reflections.[6] The present paper presents some technical details on the development of the HAC and shows recent experimental results for multiferroic $\mathrm{TbMnO}_{3}$ and $\mathrm{CuFeO}_{2}$.

\section{Development of hybrid anvil cell \\ 2.1 Hybrid anvil cell}

The HAC was originally developed by Dr. Osakabe at JRR-3 Tokai for neutron diffraction experiments.[3,4] The cell, made of beryllium copper alloy, is a kind of opposite-anvil-type cell. Two materials are used for the anvil in the HAC. A normal sapphire (or $\mathrm{SiC}$ ) single crystal is used on one side of the anvil, and WC (Co binder) is used on the other side. The sapphire anvil is supported by MP35N alloy. When we use the HAC for SNP experiments, a non-magnetic diamond composite ( $\mathrm{SiC}$ binder) or WC (Ni binder) is used instead of the ferromagnetic WC (Co binder) to avoid depolarization of polarized neutrons (Fig. 1(a)). The culet diameters are $\sim 2.7$ and $\sim 4.2 \mathrm{~mm}$ for maximum pressures of up to 10 and 3.0 GPa, respectively. We can measure pressure during loading using the ruby fluorescence method since the anvil is transparent on one side. A $0.25-\mathrm{mm}$-thick aluminum alloy (A2017) plate with a 1.0-2.0 mm hole is used for the gasket. Typical sample sizes are $0.6 \times 0.6 \times$ 0.2 and $1.2 \times 1.2 \times 0.2 \mathrm{~mm}^{3}$ for high- and low-pressure setups, respectively.

2.2 Multi-extreme condition 

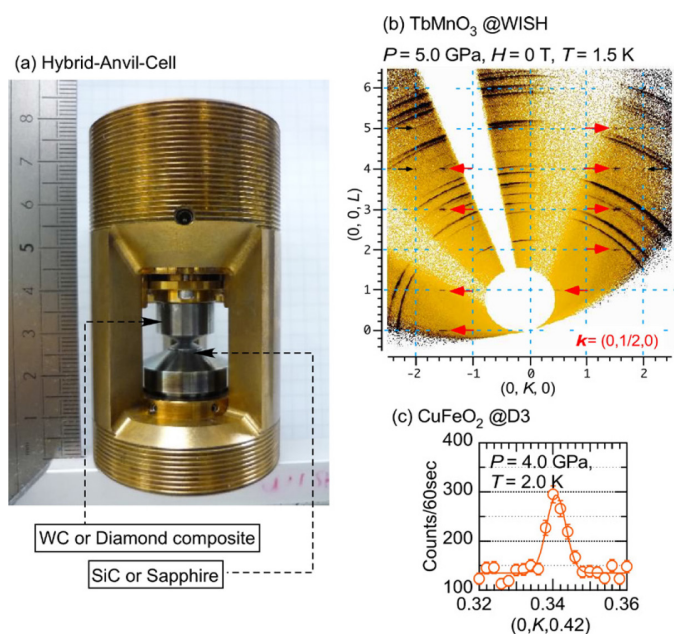

Fig. 1: (a)Photograph of the Hybrid-Anvil-Cell made of $\mathrm{Cu}-\mathrm{Be}$ alloy, and WC (or diamond composite) and $\mathrm{SiC}$ (or sapphire) anvils.[3,4] (b)Typical diffraction intensity map measured at $1.5 \mathrm{~K}, 5.0 \mathrm{GPa}$ and $0 \mathrm{~T}$ for $\mathrm{TbMnO}_{3}$ single crystal on WISH diffractometer at ISIS.[9] Big and small arrows indicate magnetic and nuclear Bragg reflections, respectively. (c)Magnetic diffraction profile observed with polarized neutrons at $2.0 \mathrm{~K}$

For standard magnetic structure analysis with unpolarized neutrons, one generally needs to collect a sufficient number of reflections beyond a scattering plane. The HAC has large opening windows of $87^{\circ}$ in the horizontal direction and -35 to $45^{\circ}$ in the vertical direction (Fig. 1(a)). Therefore, it can be effectively used in combination with large-coverage neutron detectors and the TOF technique with a wide-energy-band white beam, such as WISH.[5] A typical diffraction intensity map is shown in Fig. 1(b). Many magnetic Bragg reflections as well as nuclear ones can be seen in a wide reciprocal lattice area even for a single exposure, which makes it possible to conduct standard magnetic structure analysis, as described below.

2.3 Spherical neutron polarimetry analysis

SNP analysis is a very powerful technique for determining complex magnetic structures.[6] Since this technique is useful even when a small number of reflections are measurable, SNP is expected to work for high-pressure experiments with a tiny sample. However, the sample space during an SNP experiment must be under zero magnetic field to avoid any depolarization of incident or scattered neutrons. Therefore, the HAC should be made of nonmagnetic materials. For the SNP experiment, we employed a nonmagnetic diamond composite (with an $\mathrm{SiC}$ binder) or $\mathrm{WC}$ with a nonmagnetic $\mathrm{Ni}$ binder as the anvil materials. Nonmagnetic materials were also carefully selected for the other parts of the cell by assessing their magnetism via low-temperature magnetization measurements. Figure 1(c) shows a typical diffraction profile for an
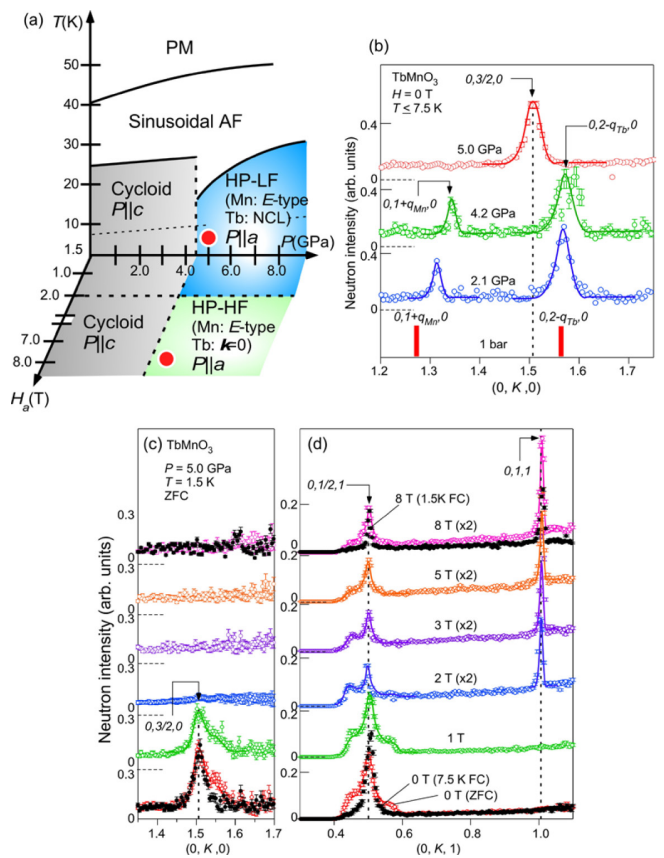

Fig. 2: (a)Magnetic phase diagram as functions of temperature, pressure and magnetic field for $\mathrm{TbMnO}_{3}$. (b)Pressure and (c,d) magnetic field dependence of diffraction patterns at low temperature for $\mathrm{TbMnO}_{3}$.[9]

incommensurate magnetic reflection at $\boldsymbol{Q}=(0,0.34,0.43)$ for $\mathrm{CuFeO}_{2}$ measured at $4.0 \mathrm{GPa}$ at the $\mathrm{D} 3$ beamline at ILL.[11] The signal-to-noise ratio for magnetic reflections is typically $\sim 1.0$. This is good enough to measure the full polarization matrix for each magnetic Bragg reflection on a reasonable timescale. However, considering the data accuracy of the observed polarization matrix elements at $4.0 \mathrm{GPa}$ in the present SNP experiment, the present case, with a sample size of $0.04 \mathrm{~mm}^{3}\left(0.4 \times 0.5 \times 0.2 \mathrm{~mm}^{3}\right)$ and a density of magnetic moment of $\sim 0.09 \mu_{\mathrm{B}} / \AA^{3}$, can be considered as being close to the limit of feasibility.

\section{Recent results}

3.1 Pressure-induced giant ferroelectricity in multiferroic $\mathrm{TbMnO}_{3}$

A pressure-induced ferroelectric polarization flop phenomenon with a drastic increase of the polarization value has been discovered in multiferroic perovskite $\mathrm{TbMnO}_{3}$.[7] The magnetic phase diagram is shown in Fig. 2(a). The pressure-induced polarization value is further increased by the application of a magnetic field, leading to a maximum polarization of $\sim 1.3 \mu \mathrm{C} / \mathrm{cm}^{2}$. Magnetic structures in high-pressure phases have not been experimentally investigated, apart from powder neutron diffraction measurements for a limited pressure range.[8] We performed a neutron diffraction experiment with the HAC at the WISH beamline on a $\mathrm{TbMnO}_{3}$ single crystal.[9] 
(a) HP-LF (5.0 GPa, 0 T, 7.5 K)

Mn: $E$-type, $\boldsymbol{k}=(0,1 / 2,0)$

Tb: NCL, $\boldsymbol{k}=(0,1 / 2,0)$

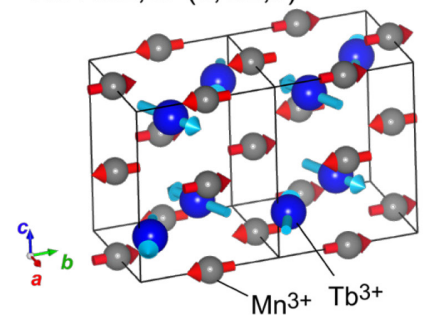

(b) HP-HF (5.0 GPa, $8 \mathrm{~T}, 1.5 \mathrm{~K})$

Mn: $E$-type, $\boldsymbol{k}=(0,1 / 2,0)$

Tb: $F_{x} C_{y}, \boldsymbol{k}=(0,0,0)$

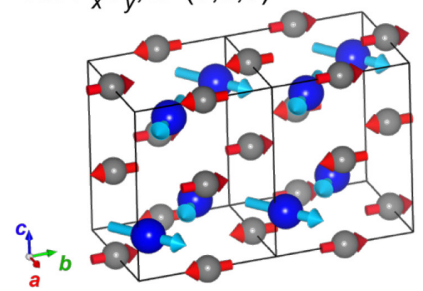

Fig. 3: The determined magnetic structures in (a)HP-LF (5.0 GPa, $0 \mathrm{~T}, 7.5 \mathrm{~K}$ ) and (b)HP-HF (5.0 $\mathrm{GPa}, 8 \mathrm{~T}, 1.5 \mathrm{~K}) .[9]$

The magnetic propagation vector (associated with the cycloid order) for the Mn spin order is $\boldsymbol{k}=(0,0.28,0)$, and the $\boldsymbol{k}$-vector for $\mathrm{Tb}$ moments is $\boldsymbol{k}=(0,0.42,0)$ at ambient pressure (Fig. 2(b)). The incommensurate orders are stable with a slight change in incommensurability up to $4.2 \mathrm{GPa}$. Above the critical pressure of $4.5 \mathrm{GPa}$, the peak position is significantly changed to be commensurate $(0,3 / 2,0)$, indicating that $\boldsymbol{k}=(0,0.5,0)$. Since the $E$-type spin configuration for $\mathrm{Mn}$ spins gives reflections on $(0,1 / 2+/-m, n)$ with only $\mathrm{n}=$ odd due to antiferrmagnetic stacking along the $c$-axis, the reflection with $\mathrm{n}=$ even originates from $\mathrm{Tb}$ ordering. When a magnetic field is applied along the $a$-axis, $\mathrm{n}=$ even $(\mathrm{Tb}$ order) disappears above a critical field of 2 $\mathrm{T}$, and instead, reflections associated with $\boldsymbol{k}=(0,0,0)$ are induced by the field. In contrast, the $E$-type $\mathrm{Mn}$ order is kept even above $2 \mathrm{~T}$ (Fig. 2(c, d)).

Magnetic structure refinements were performed in the high-pressure/low-field (HP-LF) phase, and the HP/high-field (HP-HF) phase. The magnetic structures, including $\mathrm{Tb}$ as well as $\mathrm{Mn}$ ordering, were determined (Fig. 3(a)). The magnetic order for Mn spins was determined to be the $E$-type structure with polar magnetic point group $2 \mathrm{~mm}$ in both phases. For $\mathrm{Tb}$ moments, non-collinear polar ordering (Fig. 3(a)) gives the best result in this refinement for the data measured at $7.5 \mathrm{~K}, 0 \mathrm{~T}$, and $5.0 \mathrm{GPa}$ in the HP-LF phase. For the HP-HF phase (1.5 K, $8 \mathrm{~T}$, and $5.0 \mathrm{GPa})$, the Tb ordering was determined to be a nonpolar $\boldsymbol{k}=(0,0,0)$ structure (Fig. 3(b)).

Therefore, we now understand the nature of the phase transitions observed in previous polarization measurements. The giant ferroelectric polarization with a magnitude on the order of $\sim 1.0 \mathrm{C} / \mathrm{cm}^{2}$ is induced by the $E$-type magnetic ordering for $\mathrm{Mn}$ spins. Moreover, by determining the additional $\mathrm{Tb}$ spin ordering, we can
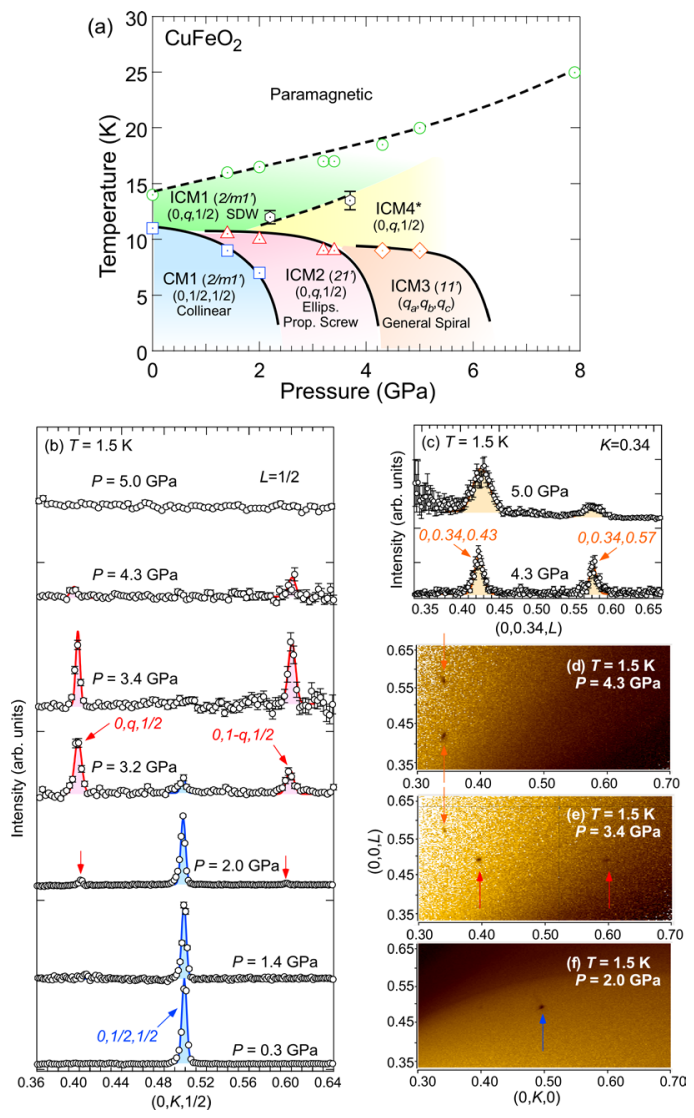

Fig. 4: (a)Magnetic phase diagram as functions of pressure and temperature for $\mathrm{CuFeO}_{2}$.[11] $(b, c)$ Typical diffraction profiles in several pressures for $\mathrm{CuFeO}_{2}$. Contour maps of neutron intensity measured at (d)4.3 GPa, (e)3.4 GPa, and (f)2.0 GPa, and 1.5 K.[10]

propose that the magnetic-field-induced polarization enhancement occurs through a mechanism in which the polarization, reduced by the polar ordering of the $\mathrm{Tb}$ moments in a zero field, is recovered through the field-induced transition from polar to nonpolar $\mathrm{Tb}$ ordering at $2 \mathrm{~T}$.

3.2 Pressure-induced nonpolar-to-polar phase transitions in delafossite $\mathrm{CuFeO}_{2}$

3.2.1 Unpolarized neutron diffraction

From unpolarized neutron diffraction and SNP experiments, $[10,11]$ we found some phase transitions from the collinear nonpolar phase to noncollinear ferroelectric phases in $\mathrm{CuFeO}_{2}$. The determined phase diagram is shown in Fig. 4(a). In addition to known phases, namely the collinear commensurate phase (CM1) and the collinear incommensurate spin-density-wave (SDW) phase (ICM1), we found several pressure-induced phases, namely ICM2, ICM3, and ICM4 phases with a noncollinear spiral magnetic structure.[10,11]

As shown in Fig. 4(b), with increasing pressure at $1.5 \mathrm{~K}$, the magnetic neutron diffraction profile along the $(0, K, L)$ reciprocal line is completely changed in terms of its peak positions, from the commensurate $(0,1 / 2,1 / 2)$ 

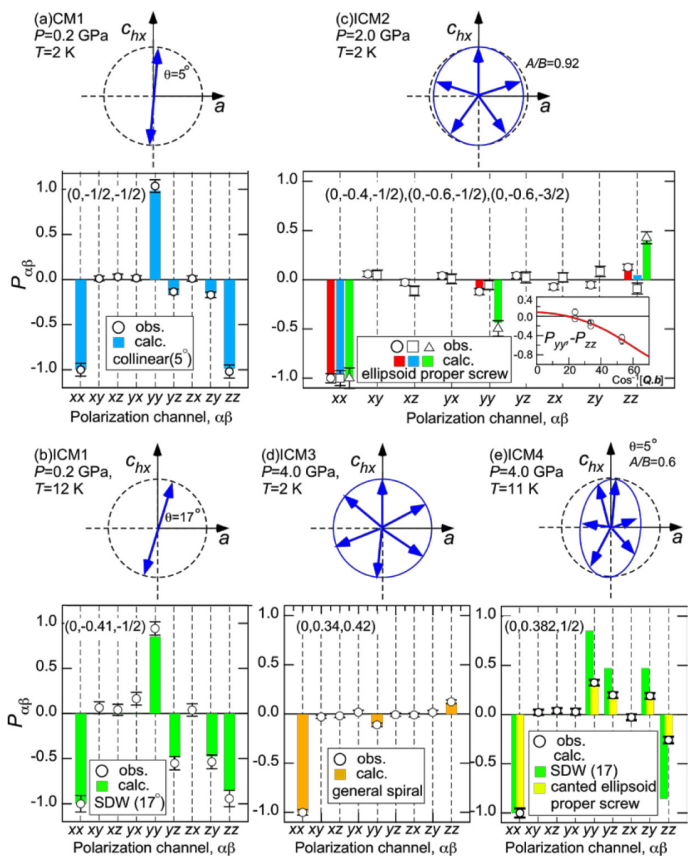

Fig. 5: Magnetic structure model projected to the monoclinic ac-plane, and comparison between observed and calculated polarization matrix elements described by eq. (1), in (a)CM1, (b)ICM1, (c)ICM2, (d)CIM3, and (e)ICM4 phases.[11]

in the CM1 phase to the incommensurate $(0, q, 1 / 2)$ with $q \sim 0.4$ in the ICM2 phase, at around $P=2.0 \mathrm{GPa}$. Further pressurizing induces another phase transition, from ICM2 to ICM3, at around $P=4.0 \mathrm{GPa}$. In the ICM3 phase, the magnetic propagation vector is changed to the general position $\boldsymbol{k}=(0,0.34,0.42)$, as shown in Fig. $4(\mathrm{c}$, d). With a further increase in pressure in the ICM3 phase, the system exhibits another phase transition to the ICM4 phase with $\boldsymbol{k}=(0, q, 1 / 2)$ and $q=0.384$ at above $P=6.0$ $\mathrm{GPa}$. The ICM4 phase can be distinguished from the ICM1 phase by the temperature-dependent or -independent $\boldsymbol{k}$-vector and the magnetic structure, namely collinear SDW in ICM1 and noncollinear spiral in ICM4, as discussed below.

3.2.2 Spherical neutron polarimetry

Although we found several pressure-induced phases under high-pressure conditions in $\mathrm{CuFeO}_{2}$ in our unpolarized neutron diffraction work, it was difficult to determine the magnetic structures, including detailed structure parameters, due to the limited number of observable magnetic reflections. In order to investigate the magnetic structures in the pressure-induced phases, an SNP analysis experiment was carried out with the non-magnetic HAC described in the previous section.

In the present SNP experiment, the single crystal was mounted with the $a$-axis vertical to provide access to the $(0, K, L)$ reflections. $\boldsymbol{P}_{\alpha \beta}$ is defined to be the polarization ratio of neutrons scattered along the $\beta(=\mathrm{x}, \mathrm{y}, \mathrm{z})$ direction when the incident neutron vector is parallel to the $\alpha(=\mathrm{x}, \mathrm{y}, \mathrm{z})$ direction. The $\mathrm{x}$-axis is parallel to the scattering vector, the $\mathrm{z}$-axis is perpendicular to the scattering plane, and the $y$-axis is orthogonal to the $x$ and $\mathrm{z}$-axes in right-handed Cartesian coordination. Assuming an ellipsoidal spiral magnetic structure with a spiral axis along a general direction (of which collinear and helical structures are special cases), we can express the $\boldsymbol{P}_{\alpha \beta}$ matrix as [11]

$$
\begin{gathered}
\boldsymbol{P}_{\alpha \beta}=\left(\begin{array}{ccc}
P_{x x} & P_{x y} & P_{x z} \\
P_{y x} & P_{y y} & P_{y z} \\
P_{z x} & P_{z y} & P_{z z}
\end{array}\right)=\left(\begin{array}{ccc}
-1 & 0 & 0 \\
P_{y x} & P_{y y} & P_{y z} \\
P_{y x} & P_{y z} & -P_{y y}
\end{array}\right) \\
P_{y x}=\mp \frac{2\left|\boldsymbol{A}_{\perp} \times \boldsymbol{B}_{\perp}\right|}{A_{\perp}^{2}+B_{\perp}^{2}} D \\
P_{y y}=\frac{A_{\perp y}^{2}-A_{\perp z}^{2}+B_{\perp y}^{2}-B_{\perp z}^{2}}{A_{\perp}^{2}+B_{\perp}^{2}} \\
P_{y z}=2 \frac{A_{\perp y} A_{\perp z}+B_{\perp y} B_{\perp z}}{A_{\perp}^{2}+B_{\perp}^{2}}
\end{gathered}
$$

$\boldsymbol{A}_{\perp}$ and $\boldsymbol{B}_{\perp}$ are spin projection vectors along the major and minor axes on the plane perpendicular to the scattering vector, respectively. $D$ is the volume faction of the spin helicity domains, $D=\left|V_{R H}-V_{L H}\right|\left|V_{R H}+V_{L H}\right|$, where $V_{R H}\left(V_{L H}\right)$ is the volume of the right(left)-handed domain. Therefore, $P_{x x}$ is always -1 for a magnetic reflection. $P_{y x}$ reflects the helicity domain ratio, and $P_{y y}$ and $P_{y z}$ reflect the magnetic structure shape projected onto the plane perpendicular to the scattering vector.

The full polarization matrix data for each magnetic phase are summarized in Fig. 5 and compared with the calculated values.[11] In the CM1 and ICM1 phases, we confirmed that the collinear SDW structures are realized with spin canting of 5 and $17^{\circ}$ from the hexagonal c-axis (Fig. 5(a,b)). In the ICM2 phase, we determined the magnetic structure to be a proper screw with a small ellipsoidal distortion ((Fig. 5(c)), which is the same structure as that induced by magnetic field/impurity substitutions. [12-16]

In the ICM3 phase, the $\boldsymbol{k}$-vector is known as the general point, $\boldsymbol{k}=\left(q_{a}, q_{b}, q_{c}\right)$ with $q_{a}=0, q_{b}=0.34$, and $q_{c}=0.42$, giving rise to symmetry reduction down to triclinic magnetic point group 11' or -11 '.[10] In the present SNP experiment, we were able to measure one magnetic Bragg reflection on $(0,0.34,0.42)$ at $4.0 \mathrm{GPa}$ and $2.0 \mathrm{~K}$ in the ICM3 phase. As shown in Fig. 5(d), we succeed in determining the magnetic structure to be the general spiral structure with spins on the monoclinic $a c$-plane. Because of the orthogonal incommensurate components along the $a$ and $c^{*}$ directions in the $\boldsymbol{k}$-vector, the general spiral structure has a proper screw modulation along the $b$-axis and a cycloid modulation along the $c^{*}$-axis.

By measuring the full polarization matrix at $4.0 \mathrm{GPa}$ and $11 \mathrm{~K}$, we found a difference in magnetic structure between $4.0 \mathrm{GPa}$ and ambient pressure at $11 \mathrm{~K}$. The measured matrix elements cannot be explained by any collinear SDW model. Therefore, the ICM4 phase was defined in the high-pressure region. We found one possible spiral structure with monoclinic symmetry in the ICM4 phase, which is an ellipsoidal proper screw with a canted major/minor axis (Fig. 5(e)). Note that there is another possible model, an ellipsoidal spiral with spins along a general direction (triclinic symmetry), which also explains the experimental data.

\section{Summary}

In conclusion, we developed the HAC for neutron diffraction experiments with polarized and unpolarized 
neutron beams. In particular, with a completely nonmagnetic anvil, we succeeded in measuring the full polarization matrix under high pressure, up to $4.0 \mathrm{GPa}$, by combining the HAC with the CRYOPAD instrument. for the first time. By using unique setups, we clarified the magnetic phases induced by pressure for multiferroic $\mathrm{CuFeO}_{2}$ and $\mathrm{TbMnO}_{3}$.[9-11] I hope that our method will encourage researchers to study novel pressure-induced physical phenomena associated with complex magnetic ordering.

Finally, I would like to acknowledge all of my collaborators listed in the three articles.[9-11] In particular, I want to acknowledge Dr. Osakabe for very helpful advice on pressurization with the HAC, and Dr. Khalyavin and Dr. Manuel for neutron experiments on WISH, and Dr. Qureshi and Dr. Chapon for SNP experiments at ILL. I would like to acknowledge the support from the JSPS based on an international collaboration research program, JSPS KAKENHI Grant No. $15 \mathrm{H} 05433$.

[1] I. N. Goncharenko, High Press. Res. 24, 193 (2004).

[2] Stefan Klotz, "Techniques in High Pressure Neutron Scattering", CRC Press.

[3] T. Osakabe, K. Kuwahara, D. Kawana, K. Iwasa, D. Kikuchi, Y. Aoki, M. Kohgi and H. Sato, JPSJ 79, 034711 (2010).

[4] H. Yamauchi, T. Osakabe, E. Matsuoka, and H. Onodera, JPSJ 81, 034715 (2012).

[5] L. C. Chapon, P. Manuel, P. G. Radaelli, C. Benson, L. Perrott, S. Ansell, N. J. Rhodes, D. Raspino, D. Duxbury, E. Spill and J. Norris, Neutron News, 22, 22 (2011).

[6] F. Tasset, Physica B 156-57, 627 (1989).

[7] T. Aoyama, K. Yamauchi, A. Iyama, S. Picozzi, K. Shimizu, and T. Kimura, Nat. Comm. 5, 4927 (2014).

[8] O. L. Makarova, I. Mirebeau, S. E. Kichanov, J. Rodriguez-Carvajal, and A. Forget, Phys. Rev. B 84, 020408 (2011).

[9] N. Terada, D. D. Khalyavin, P. Manuel, T. Osakabe et al., Phys. Rev. B 93 081104(R) (2016).

[10] N. Terada, D. D. Khalyavin, P. Manuel, T. Osakabe et al., Phys. Rev. B 89 220403(R) (2014).

[11] N. Terada, N. Qureshi, L. C. Chapon and T. Osakabe, Nat. Commun. 9, 4368 (2018).

[12] N. Terada, J. Phys.: Condens. Matt. 26, 543202 (2014).

[13] T. Kimura, J. C. Lashley and A. P. Ramirez, Phys. Rev. B 73, 220401(R) (2006).

[14] S. Seki, Y. Yamasaki, Y. Shiomi, S. Iguchi, Y. Onose and Y. Tokura, Phys. Rev. B 75, 100403(R) (2007).

[15] N. Terada, T. Nakajima, S. Mitsuda, H. Kitazawa, K. Kaneko and N. Metoki, Phys. Rev. B 78, 014101 (2008).

[16] T. Nakajima, S. Mitsuda, K. Takahashi, M. Yamano, K. Masuda, H. Yamazaki K. Prokes, K. Kiefer, S. Gerischer, N. Terada H. Kitazawa, M. Matauda, K. Kakurai, H. Kimura, Y. Noda, M. Soda, M. Matsuura, and K. Hirota Phys. Rev. B 79, 214423 (2009).

(Received September 11, 2018; Accepted November 20, 2018; Published Online February 1, 2019) 